\title{
Severe asthma associated with myasthenia gravis
}

\author{
Natasha Satkunam MSc ${ }^{1}$, Zaeem A Siddiqi MD PhD², Dilini Vethanayagam MD FRCPC ${ }^{2}$
}

N Satkunam, ZA Siddiqi, D Vethanayagam. Severe asthma associated with myasthenia gravis. Can Respir J 2014;21(1):e1-e3.

Severe asthma constitutes a subgroup of approximately $10 \%$ of all asthma cases. Approximately one-half of these individuals have a refractory form of the disease in which atopy and T-helper cell 2-skewed immunological response may not be as closely linked to the disease as in other phenotypes of asthma. This suggests that not all asthma is explained by a T-helper cell 2-skewed immunological response, and that other immunological mechanisms may be important in this category of nonatopic asthma. The authors present a case involving a 55-year-old Caucasian man with nonatopic, adult-onset asthma, nonsteroidal anti-inflammatory drug sensitivity and idiopathic urticaria. This individual presented two years following his initial asthma diagnosis with diplopia and mild ptosis, and was subsequently diagnosed with seropositive myasthenia gravis.

Key Words: Asthma; Autoimmune; Myasthenia gravis, Nonatopic; Severe asthma

\section{Learning objectives}

- To recognize that the underlying pathology of severe, nonatopic asthma may be related to an as yet poorly elucidated autoimmune process.

- To recognize that many autoimmune processes can coexist and can account for an individual's dyspnea external to an airways disorder.

CanMeds Competency: Medical Expert

Pretest

- What is the relationship between severe asthma (SA) and myasthenia gravis $(\mathrm{MG})$ ?

A sthma is a chronic inflammatory disorder of the airways that is often characterized as T-helper cell (TH) 2-mediated immune dysregulation $(1,2)$. A significant proportion of individuals with SA $(3,4)$, as defined by the American Thoracic Society and European Respiratory Society, appear to be nonatopic according to allergen skin tests $(5,6)$. The presence of a TH2-skewed immune response is debated in this latter group because it has been suggested that the underlying pathology may be due to local airway-specific responses or responses to uncommon and untested allergens (7). Recent work by Tedeschi et al (8) showed that $58 \%$ of nonatopic individuals with asthma have positive autologous serum skin test (ASST) results that were not noted in any of the healthy controls nor their atopic counterparts. In a small proportion of these nonatopic individuals with asthma and positive ASST, histamine-releasing autoantibodies were detected. In another recent study (9), immunoglobulin $G$ autoantibodies against bronchial epithelial cells were implicated in the pathogenesis and maintenance of inflammation in individuals with nonatopic asthma. Thus, in these nonatopic individuals with SA, the immunobiology has been less clearly delineated, and there may be evidence of underlying autoimmune disease processes.

\section{Un asthme sévère associé à une myasthénie grave}

L'asthme sévère représente un sous-groupe d'environ $10 \%$ de tous les cas d'asthme. Environ la moitié d'entre eux ont une forme réfractaire de la maladie, pour laquelle l'atopie et la réponse immunologique faussée par les lymphocytes $T$ auxiliaires 2 ne seraient pas aussi liées à la maladie que dans les autres phénotypes d'asthme. D'après cette constatation, tous les cas d'asthme ne s'expliquent pas par une réponse immunologique faussée par les lymphocytes $\mathrm{T}$ auxiliaires 2, et d'autres mécanismes immunologiques pourraient avoir de l'importance dans cette catégorie d'asthme non atopique. Les auteurs présentent le cas d'un homme blanc de 55 ans ayant un asthme non atopique apparu à l'âge adulte, une sensibilité aux antiinflammatoires non stéroïdiens et une urticaire idiopathique. Deux ans après son diagnostic initial d'asthme, il a consulté en raison d'une diplopie et d'une ptose bénigne, et on lui a ensuite diagnostiqué une myasthénie grave séropositive.

MG is a neuromuscular disorder characterized by fluctuating muscle weakness and fatigability due to antibody-mediated autoimmune processes at the neuromuscular junction (10). MG patients can be broadly split into two groups: those who are acetylcholine receptor (AchR) antibody positive and those who are AchR antibody negative. MG is strongly linked with thymoma, but has also been associated with other autoimmune diseases $(10,11)$. We present a case involving a patient with nonatopic SA on the background of other immunological conditions, specifically seropositive MG and idiopathic urticaria.

\section{CASE PRESENTATION}

A 55-year-old Caucasian man with adult-onset asthma initially presented with a one-year history of dyspnea. He had associated chronic rhinosinusitis with nasal polyposis. He was an exsmoker with a 50 packyear history but had quit one year before his presentation. Over time, airway physiology showed fixed airflow limitation. Spirometric values on initial assessment showed a forced expiratory volume in $1 \mathrm{~s}\left(\mathrm{FEV}_{1}\right)$ of $1.67 \mathrm{~L}$ (41\% predicted) and a forced vital capacity (FVC) of $3.08 \mathrm{~L}$ (61\% predicted), with an $\mathrm{FEV}_{1} / \mathrm{FVC}$ ratio of 54 . He demonstrated a $42 \%$ improvement in $\mathrm{FEV}_{1}$ to $2.36 \mathrm{~L}$ and a $21 \%$ improvement in FVC to $3.73 \mathrm{~L}$ post-beta-agonist bronchodilation (2). Assessment three years later showed an $\mathrm{FEV}_{1}$ of $2.24 \mathrm{~L}$ (58\% predicted) and an FVC of $3.72 \mathrm{~L}$ ( $78 \%$ predicted). There was a $10 \%$ improvement post-betaagonist bronchodilation on that occasion, which was tested after having held his inhaled medications that morning. Lung volumes were normal. Diffusion impairment was noted on early assessment; however, repeat testing showed normalization. High-resolution computed tomography of his chest did not reveal any evidence of emphysematous changes, bronchiectasis or interstitial lung disease. He had no current or history of pulmonary vascular disease. Sputum cell counts over a two-year period showed variable airway neutrophilia and mixed granulocyte inflammation. This patient suffered from nonsteroidal antiinflammatory drug sensitivity, including one episode of anaphylaxis associated with the use of ibuprofen around his initial presentation. and Dentistry, University of Alberta, Edmonton, Alberta

Correspondence: Dr Dilini Vethanayagam, Room 3-105C, Clincal Sciences Building, University of Alberta, 11350-83 Avenue, Edmonton, Alberta T6G 2J2. Telephone 780-492-5879, fax 780-492-4483, e-mail dilini@ualberta.ca 
His respiratory symptoms were also triggered by exertion, stress, fumes, fragrances and cigarette smoke, but not by outdoor pollens or furred animals. Allergen skin tests did not show any evidence of atopy on two separate occasions. At the time of the initial evaluation, he experienced episodes of dyspnea multiple times daily and had marked exercise limitation. He was placed on inhaled corticosteroids and a long-acting beta-agonist in the form of budesonide/formoterol $(200 \mu \mathrm{g} / 6 \mu \mathrm{g}$ two inhalations twice daily) and a beta 2 -agonist reliever on an as-needed basis. He was prescribed a therapeutic trial of a systemic steroid (prednisone $30 \mathrm{mg}$ daily); however, after five days, he experienced hip pain and was subsequently diagnosed with steroid-induced avascular necrosis of the hip. He also developed urticaria of increasing frequency and severity, which through repeated evaluations was believed to be idiopathic and for which he was placed on cetirizine $10 \mathrm{mg}$ daily. He was found to have an elevated antithyroid peroxidase antibody level $(118 \mathrm{kU} / \mathrm{L}$ [upper limit of normal $100 \mathrm{kU} / \mathrm{L}$ ]) despite normal thyroid studies, and was prescribed a therapeutic trial of thyroid replacement therapy with levothyroxine $125 \mu \mathrm{g}$ per day (11-13). This resulted in resolution of his urticaria; however, when this medication was removed for a short period of time one year later, the urticaria recurred.

Four years after initial presentation, he developed diplopia and mild ptosis of two weeks' duration, which prompted a neurological evaluation. His clinical examination, nerve conduction studies and single-fibre electromyography were suggestive of a neuromuscular transmission disorder. Subsequently, his serum AchR antibody levels were found to be elevated $(18.80 \mathrm{nmol} / \mathrm{L}$ [normal $<0.25 \mathrm{nmol} / \mathrm{L}])$, confirming the diagnosis of seropositive MG with predominantly ocular presentation. He was treated with a course of intravenous immunoglobulin followed by azathioprine, which resulted in remission of his myasthenic symptoms. However, there was no change noted in his respiratory symptoms. He continues to be managed for his asthma and idiopathic urticaria through the Edmonton Regional Severe Asthma Clinic (Edmonton, Alberta).

\section{DISCUSSION}

Our patient's history and presentation suggest that he has an underlying immune dysregulation that predisposes him to multiple autoimmune disorders. He had high levels of antithyroid peroxidase antibodies, which has been associated with both of his conditions chronic idiopathic urticaria and ocular MG (11-14). In fact, patients with ocular MG, as opposed to generalized MG, are more likely to have autoimmune thyroid disease, which suggests a common underlying genetic predisposition and immune profile (11). While an ASST has not been performed in our patient, it has been found that individuals with nonatopic asthma are more likely to test positive on ASST than individuals with atopic asthma. Furthermore, a positive ASST has also been associated with chronic urticaria and nonsteroidal antiinflammatory drug sensitivity, conditions also found in our patient (8).

This patient represents one of two cases linking SA and MG that are currently being followed through the Edmonton Regional Severe Asthma Clinic. The second patient, who is now an adult, had longstanding childhood-onset atopic SA, significant environmental allergies (for which she previously received allergen immunotherapy), ongoing allergic conjunctivitis and common variable immunodeficiency. She was diagnosed with seronegative generalized MG. Three other patients in the Myasthenia Clinic based at the same location also have concomitant - but not severe - asthma.

Collectively, this suggests that a neuromuscular weakness should be screened for as part of the systematic evaluation of individuals considered to have refractory SA not responsive to airway-focused treatments. This should include objective neuromuscular evaluations such as nerve conduction studies and electromyography. The purpose of this screening is to elucidate the etiology of the common symptom of dyspnea, which has a broad differential diagnosis. Dyspnea in an asthma patient may be due to airway and nonairway processes including MG. If the latter were not evaluated, this may result in overtreatment for the individual from an airways perspective alone, and missed or delayed diagnosis of an important neuromuscular process.

There is recent evidence of an association between asthma and a non-TH2-skewed response, with different cytokines being implicated in its pathogenesis (15). In addition, there are hypotheses regarding specific immunoglobulin E production against infectious antigens, which could be responsible for both inception and aggravation of nonallergic asthma (16). Asthma has also been correlated to a variety of autoimmune disorders. Epidemiological studies have revealed a link between asthma and autoimmunity in type I diabetes, and airway hyper-responsiveness likely secondary to structural damage and inflammatory infiltration, has been noted in patients with autoimmune conditions such as rheumatoid arthritis, systemic sclerosis and Sjögren syndrome (15). We propose that SA and non-TH2-related immunological disorders, such as $\mathrm{MG}$, can coexist, and that an association should be studied further. We postulate that underlying autoimmune disorders in some individuals with SA who have persistent dyspnea as their primary respiratory symptom not responding to traditional asthma therapies should be screened for neuromuscular disease. In fact, a clinical model that incorporates a broader multisystem assessment and ongoing evaluation should be used for a chronic airways disease, such as asthma, such that patients who do not respond to targeted airways-specific therapeutic regimens will be assessed for coexisting conditions that include neuromuscular disorders.

\section{Post-test}

- What is the relationship between SA and MG?

MG is an autoimmune condition that has been associated with other autoimmune conditions. In nonatopic SA patients, the two conditions may coexist, and the underlying pathology may relate to a broader autoimmune process which, as yet, has not been elucidated. MG is a condition that should be considered in cases of nonatopic SA that do not respond to treatment, especially in patients with other autoimmune diseases.

ACKNOWLEDGEMENTS: The authors thank Ms Iris de Guzman for assistance in this study.

\section{REFERENCES}

1. Global Strategy for Asthma Management and Prevention. Global Initiative for Asthma (GINA) 2010. <www.nejm.org/toc/ nejm/326/5/> (Accessed November 27, 2011).

2. Lougheed MD, Lemiere C, Ducharme FM, et al. Canadian Thoracic Society 2012 guideline update: Diagnosis and management of asthma in preschoolers, children and adults. Canadian Thoracic Society Asthma Clinical Assembly. Can Respir J 2012;19:127-64.

3. American Thoracic Society. Proceedings of the ATS workshop on refractory asthma. Am J Respir Crit Care Med 2000;162:2341-51.

4. Bousquet J, Mantzouranis E, Cruz AA, et al. Uniform definition of asthma severity, control, and exacerbations: Document presented for the World Health Organization Consultation on Severe Asthma. J Allergy Clin Immunol 2010;126:926-38.

5. Moore WC, Bleecker ER, Curran-Everett D, et al. Characterization of the severe asthma phenotype by the National Heart, Lung, and Blood Institute's Severe Asthma Research Program. J Allergy Clin Immunol 2007;119:405-13.

6. Moore WC, Meyers DA, Wenzel SE, et al. Identification of asthma phenotypes using cluster analysis in the Severe Asthma Research Program. Am J Respir Crit Care Med 2010;181:315-23.

7. Humbert M, Menz G, Ying S, et al. The immunopathology of extrinsic (atopic) and intrinsic (non-atopic) asthma: More similarities than differences. Rev Immunol Today 1999;20:528-33.

8. Tedeschi A, Comi AL, Lorini M, Tosini C, Miadonna A. Autologous serum skin test reactivity in patients with non-allergic asthma. Clin Exp Allergy 2005;35:849-53.

9. Kwon B, Choi GS, Park HS, Nahm DH, Lee HA, Ye YM. Increased $\mathrm{IgG}$ antibody-induced cytotoxicity against airway epithelial cells in patients with nonallergic asthma. J Clin Immunol 2009;29:517-23. 
10. Vincent A, Palace J, Hilton-Jones D. Myasthenia gravis. Lancet 2001;357:2122-8.

11. Marinó M, Ricciardi R, Pinchera A, et al. Mild clinical expression of myasthenia gravis associated with autoimmune thyroid diseases. J Clin Endocrinol Met 1997;82:438-43.

12. Kiyici S, Gul OO, Baskan EB, et al. Effect of levothyroxine treatment on clinical symptoms and serum cytokine levels in euthyroid patients with chronic idiopathic urticaria and thyroid autoimmunity. Clin Exp Dermatol 2010;35:603-7.

13. Sheikh J, Saini SS, Kulczycki A Jr, Dreskin SC. Urticaria and Angioedema Committee of the American Academy of Allergy,
Asthma \& Immunology. A survey of allergists regarding the association of thyroid autoimmunity with chronic urticaria. J Allergy Clin Immunol 2009;123:1173-5.

14. Pal J, Rozsa C, Komoly S, Illes Z. Clinical and biological heterogeneity of autoimmune myasthenia gravis. J Neuroimmunol 2011;231:43-54.

15. Souwer Y, Szegedi K, Kapsenberg ML, deJong EC. IL-17 and IL-22 in atopic allergic disease. Curr Opin Immunol 2010;22:821-6.

16. Tedeschi A, Asero R. Asthma and autoimmunity: A complex but intriguing relation. Expert Rev Clin Immunol 2008;4:767-76. 


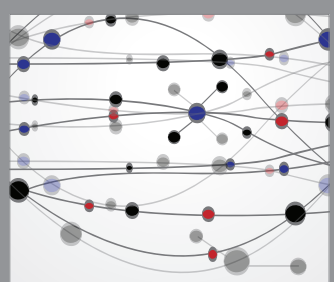

The Scientific World Journal
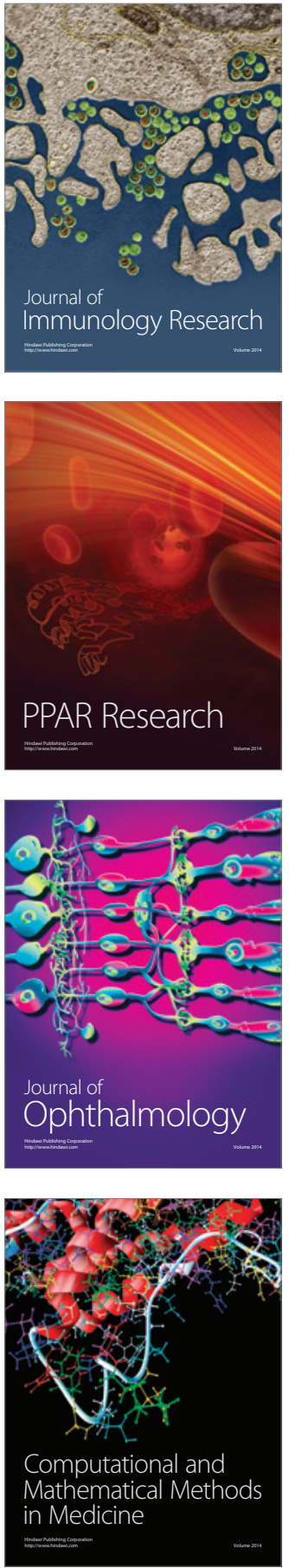

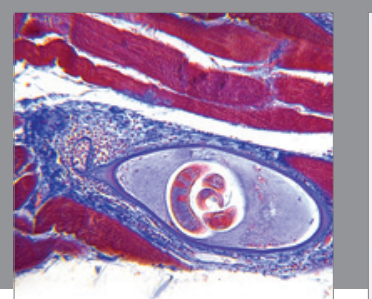

Gastroenterology Research and Practice

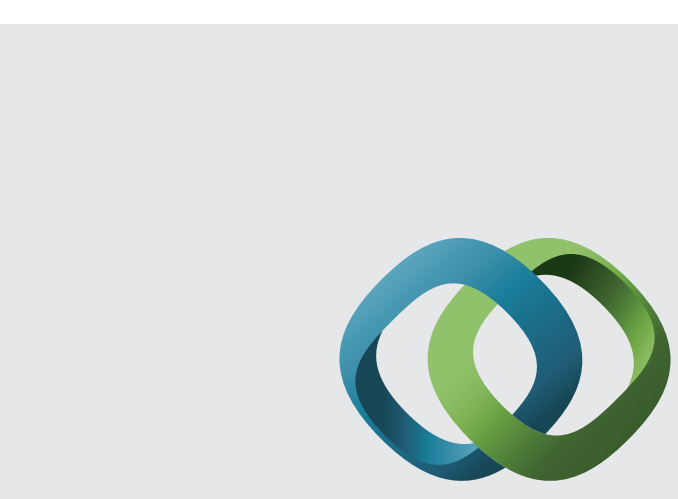

\section{Hindawi}

Submit your manuscripts at

http://www.hindawi.com
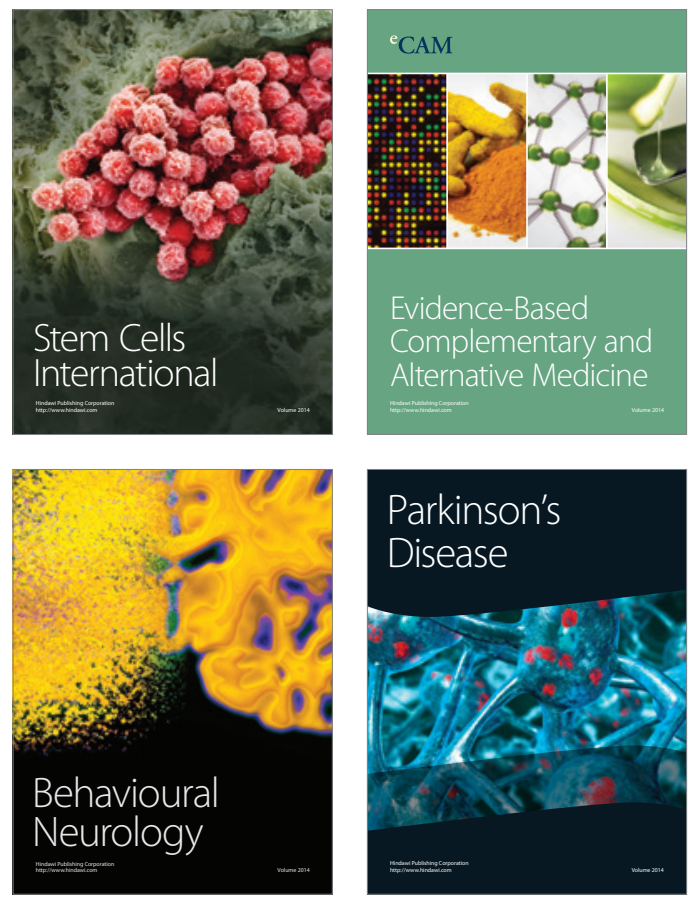
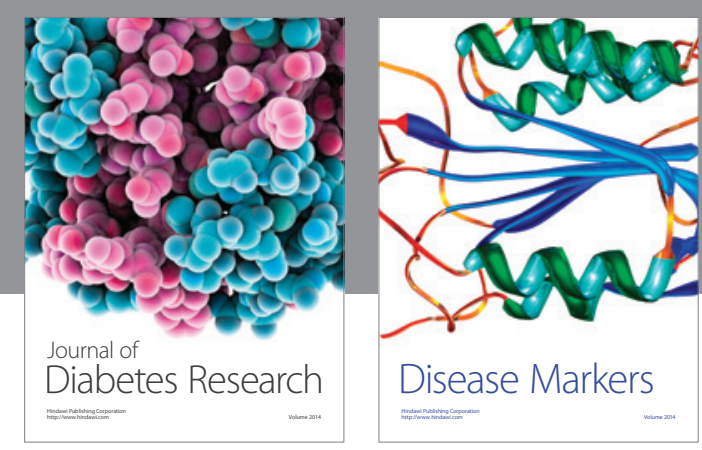

Disease Markers
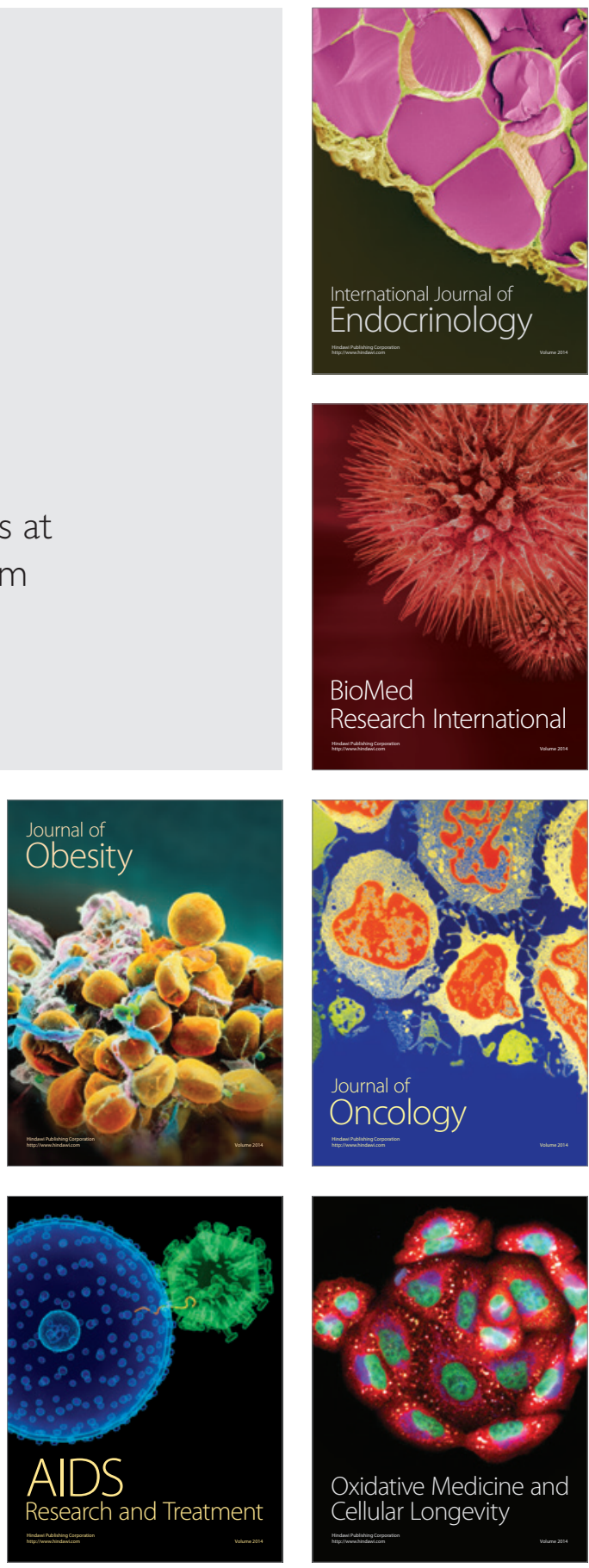